\title{
Motion-Compensated Frame Rate Up-Conversion Based on Block Matching Algorithm
}

\author{
Shinya Fujiwara and Akira Taguchi \\ Department of Electrical and Electronic Engineering \\ Musashi Institute of Technology \\ Tamazutsumi, Setagaya-ku, Tokyo 158-8557, JAPAN
}

\begin{abstract}
In this paper, we propose a frame rate up-conversion (FRUC) method using the motion compensation (MC) based on block matching algorithm (BMA). The property of the BMA is changed by the size of block. It is desirable for small moving object to set block size small. On the other hand, we would like to set the block size large in the global motion region. Therefore, the block size is decided depend on the property of the local region of frame image. In this paper, we present a novel FRUC technique based on the motion compensated interpolation with multi-size blocks. At first, the frame image is divided into $8 \times 8$ blocks and motion vectors are estimated for each $8 \times 8$ block. Next, the similar motion blocks are merged into one block, up to $32 \times 32$ block. Thus he proposed method reduces block artifacts and realizes the clear interpolation of moving objects regardless of the size of those.
\end{abstract}

Keywords - frame rate up-conversion, motion estimation, motion-compensated interpolation, multi-size block

\section{INTRODUCTION}

Frame rate up-conversion (FRUC) is necessary for conversion between various display formats with different frame rates. Generally, simple FRUC algorithms such as frame repetition and linear interpolation by temporal filtering have been used. But, these simple algorithms cause motion jerkiness into motion portrayal and blurring of moving objects. To reduce the motion jerkiness, several motion-compensated FRUC (MC-FRUC) algorithms have been developed [1]-[3]. However, MC-FRUC algorithms introduce blocking artifacts whenever the motion estimation (ME) is incorrect.

In order to achieve high-quality conversion, the MC-FRUC algorithms use a special kind of ME [4],[5]. The ME used in [4] utilizes regularity of motion vectors in neighboring blocks to prevent degradation of picture quality. Furthermore, overlapped block motion compensations are used, which provide improved prediction accuracy as well as better subjective quality in video coding at the expense of increased computational complexity [6].

This pap er presents a novel FRUC technique based on the motion compensated interpolation with multi-size blocks (MCFMSB). The proposed method is based on the simple $\mathrm{ME} / \mathrm{MC}$ between two adjacent frames. At first, motion vectors of $8 \times 8$ size blocks are estimated. Usually, even in still regions, the motion vector is not equal to zero. Thus, each $8 \times 8$ region is judged whether still or move by threshold operation. Then, if the region is judged still, we force the motion vector to zero of the region. Global motion regions and local motion regions are mixed in each frame. In the global motion regions, it is desirable that the block size of ME/MC is larger. In other motion regions, it is desirable that the block size of $\mathrm{ME} / \mathrm{MC}$ is fit to that of moving objects. In the proposed method, we merge four $8 \times 8$ blocks into a $16 \times 16$ block if necessary. Furthermore, we merge four 16x16 blocks into a $32 \times 32$ block if necessary. The proposed method reduces block artifacts, since similar motion blocks are merged into one block. Moreover moving objects are interpolated clearly, since even if the moving object is located into multi $8 \times 8$-blocks, these blocks are merged into one block.

Section II presents details of the proposed method, including the conventional ME. In Section III, experimental results are given and discussed. Finally Section IV concludes this paper.

\section{PROPOSED MC-FRUC}

Figure 1 shows the block diagram of the proposed MC-FRUC scheme. The first step in the proposed method is to estimate the motion. The proposed method is based on block matching algorithm (BMA) with $8 \times 8$-block. BMA has been widely used to determine the motion vectors between two adjacent frames. However, the accuracy of BMA depends on the block size. If block size is small, the motion vector, which is calculated by the still region, is not equal to zero. Furthermore, the accuracy of motion vector, which is given by a small block, is low. The threshold procedure and the vector median filter perform the motion vectors of $8 \times 8$-block. Moreover, we would like to change the block size of 


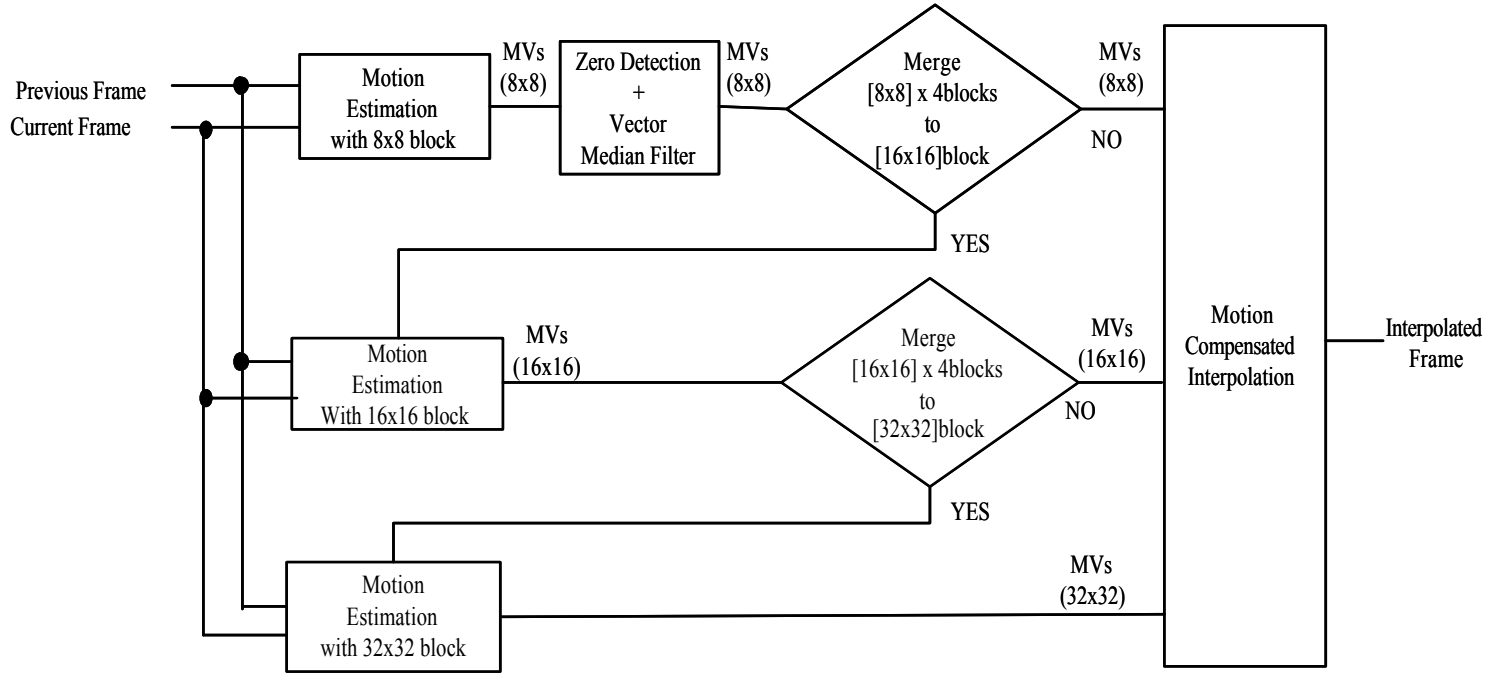

Fig.1 The block diagram of the proposed MC-FRUC method

BMA depend on the property of motion and the size of moving objects. In the global motion regions, it is desirable that the block size of BMA is larger. On the other hand, in the local motion regions, it is desirable that the block size of BMA is fit to that of the moving object. We investigate the property of local motion characteristic by motion vectors of the $8 \times 8$-block and merge four $8 \times 8$ blocks into a $16 \times 16$ block if recessary. Furthermore, we merge four merged 16x16 blocks into a $32 \times 32$ block if necessary.

We explain the details of the procedure of the proposed method (see Fig.1) as follows.

\section{A. Motion Estimation with $8 x 8$-block}

The proposed MC-FRUC method is utilized the BMA with multi-block sizes. At the first, the motion vector is estimated based on BMA with $8 \times 8$-block. Let two adjacent frame images be denoted by $f(i, j, k-1)$ and $f(i, j, k)$, where $(i, j)$ and $k$ are the spatial and time domain indices, respectively. The frame image is divided into $8 \times 8$ blocks. The motion vector of $(m, n)^{\text {th }}$ block is obtained by

$$
\begin{gathered}
\mathbf{v}_{m, n}=\arg \min _{\mathbf{v}_{m, n} \in \mathbf{S}}\left\{J\left(\mathbf{v}_{m, n}\right)\right\} \\
J\left(\mathbf{v}_{m, n}\right)=\sum_{i=0}^{7} \sum_{j=0}^{7} \mid f\left(8(m-1)+i+v_{m, n}^{H}, 8(n-1)+j+v_{m, n}^{V}, k-1\right) \\
\quad-f(8(m-1)+i, 8(n-1)+j, k) \mid
\end{gathered}
$$

where $J$ is the sum of absolute difference (SAD) and $\mathbf{S}$ denotes the search range.

\section{A. Zero Detection and Vector Median Filtering}

In the case of motion vector estimation on BMA with small size block, false motion vector is often derived. For example, even if the block is located in the still region, the motion vector is not estimated zero from the effect of the additive noise or filiker. Thus, we introduce two processes in order to improve the accuracy of the motion vector.

\section{(a) Zero Detection}

We compare $J\left(\mathbf{v}_{m, n}\right)$ and $J(0)$. If the difference between two values is less than $\mu$, the block is judged located in the still region. Then, motion vector $\mathbf{v}_{m, n}$ is set to zero.

\section{(b) Vector Median Filtering}

The vector median filter with $3 \times 3$ is performed for the motion vector $\mathbf{v}_{m, n}$. The output of the vector median filter $\hat{\mathbf{v}}_{m, n}$ is defined by

$$
\hat{\mathbf{v}}_{m, n}=\arg \min _{(k, l) \in W} \sum_{(i, j) \in W}\left\|\mathbf{v}_{m+k, n+l}-\mathbf{v}_{m+i, n+j}\right\|
$$

where $W$ denotes the filter window.

The estimated motion vectors with $8 \times 8$-block are unreliable. In order to improve the accuracy of the motion vector, the motion vector is adjusted using the motion vectors of the surrounding blocks. In Ref.[3], the weighted average filtering is utilized, and in this paper, the vector median filtering is utilized for the adjustment of the motion vectors.

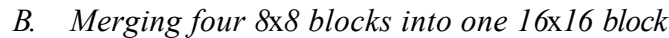

Generally, global motion and local motion are mixed in the same frame image. In the global motion region, it is good that the block size of BMA is large. On the other 
hand, in the local motion region, the block size is depended on the size of moving objects. It is necessary to decide the block size of BMA depend on the location $(i, j)$ in frame image. In this step, we merge four $8 \times 8$ blocks into one $16 \times 16$ block, if four motion vectors are similar. The following two distances measure the degree of similarity for four motion vectors.

\section{(a) Direction Distance}

We check the angle between any two-motion vectors. If all angles are less than the threshold value $\theta_{8}$, we go to next check, otherwise, four $8 \times 8$ blocks cannot be merged into one $16 \times 16$ block.

(b) $L_{2}$ Norm

We check the distance between any two-motion vectors based on $\mathrm{L}_{2}$ norm. If all distances are less than $\varepsilon_{8}$, four $8 \times 8$ blocks are merged into $16 \times 16$ block, otherwise, these blocks cannot be merged.

\section{Motion Estimation with 16x16-block}

Focus only merged blocks in the former step; we re-estimate the motion vector of these blocks with 16x16-bock.

\section{E. Merging four $16 \times 16$ blocks into one 32x32 block}

If the four 16x16 blocks are connected vertical and horizontal directions, we check whether these four blocks are merged into $32 \times 32$ block or not. In this case, we also check the angle and distance between any two-motion vectors. If all angles are less than $\theta_{16}$ and all distances are less than $\varepsilon_{16}$, four $16 \times 16$ blocks are merged into $32 \times 32$ block.

\section{F. Motion Estimation with 32x32-block}

Focus only merged blocks in the former step, we re-estimate the motion vector of these blocks with $32 \times 32$-bock.

\section{G. Motion Compensated Interpolation}

Once motion vectors with multi block sizes are determined, this motion information is utilized in the motion compensated interpolation. If the interpolated frame image exists temporally halfway between adjacent frames, the interpolated frame image is given by

$$
f\left(i, j, k-\frac{1}{2}\right)=\frac{f\left(i+\frac{v_{i}}{2}, j+\frac{v_{j}}{2}, k-1\right)+f\left(i-\frac{v_{i}}{2}, j-\frac{v_{j}}{2}, k\right)}{2}
$$

where $v_{\mathrm{i}}$ and $v_{\mathrm{j}}$ are elements of the motion vector from $8 \times 8$-block or $16 \times 16$-block or $32 \times 32$-block. Since ME is performed by the sub-block, it sometimes happens that multiple blocks are overlapped one another in the
Table 1 Average PSNR of the estimated frame images

\begin{tabular}{|l|r|r|r|r|}
\hline \multicolumn{1}{|c|}{$[\mathrm{dB}]$} & Proposed & $8 \times 8$ block & $16 \times 16$ block & $32 \times 32$ block \\
\hline Hall monitor & 37.410 & 36.425 & 36.965 & 36.972 \\
\hline Foreman & 29.826 & 29.358 & 29.572 & 29.637 \\
\hline Football & 21.668 & 21.306 & 21.521 & 21.359 \\
\hline
\end{tabular}

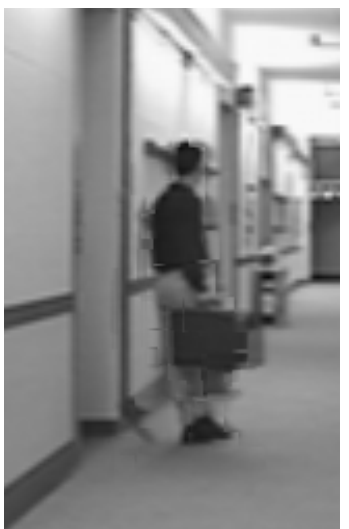

(a) Proposed

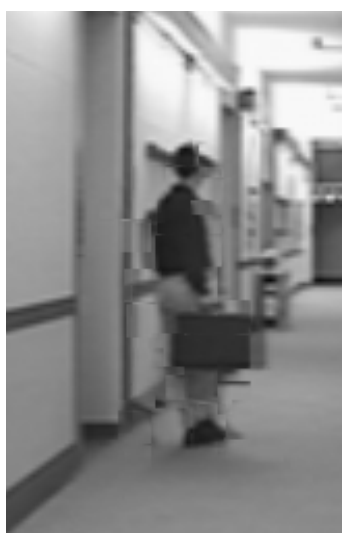

(c) 16x16-block

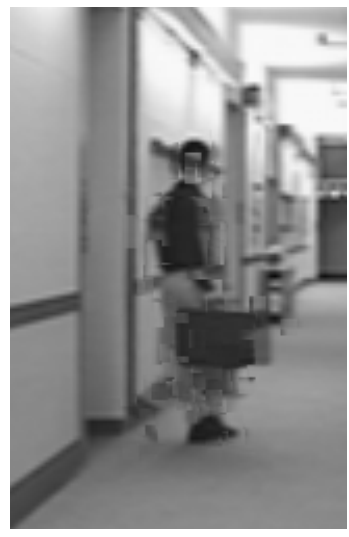

(b) $8 \times 8$-block

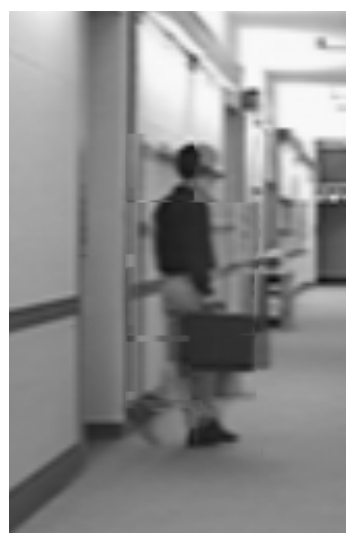

(d) $32 \times 32$-block
Fig.2 The estimated frame of each method (Hall monitor ; frameNo.17)

interpolated frame image. In this case we calculate the average of those blocks. On the other hand, if the pixel value of the interpolated frame image is not given by equation (3), we set the average value $(f(i, j, k-1)+$ $f(i, j, k)) / 2$ to the pixel value.

\section{EXPERIMENTAL RESULTS}

We prepare three test sequences (Hall monitor, Foreman, Football), which are classed in Ref. [7]. Hall monitor has low spatial detail and the low amount of movement. On the other hand, Football has high spatial detail and the high amount of movement. Foreman has 
the property between Hall monitor and Football. These sequences are constructed by 50 frames (i.e., No.0 No.49). And the size of each frame is $352 \times 288$. We pull out odd number of frame images. We estimate the odd number of frame images from even number of frame images by using the FRUC methods. The proposed MC-FRUC method compare to the MC-FRUC with fixed block size. The search range is set to \pm 16 .

It is necessary to decide the parameters of the proposed method. We use a lot of test sequences for deciding the parameters. The suit able value of the parameter $\mu$ of the "zero detection" is 190 . We also investigate of the suitable value of the parameters $\left(\theta_{8}, \varepsilon_{8}\right)$ and $\left(\theta_{16}, \varepsilon_{16}\right)$ and obtain $\left(\theta_{8}, \varepsilon_{8}\right)=\left(30^{\circ}, 4\right)$ and $\left(\theta_{16}, \varepsilon_{16}\right)=\left(30^{\circ}, 3\right)$.

Table 1 shows the average PSNR of the estimated frame (i.e., odd number of frame images). We can understand that the proposed method shows best results for all image sequences. The standard deviation of PSNR of each sequences is almost same for all methods.

Figure 2 is an example of the estimated results. Figure 2(a) shows the result of propose method. Figure 2(b)-(d) show the results of the MC-FRUC with $8 \times 8,16 \times 16$, and $32 \times 32$, respectively. The block artifacts on block boundaries are introduced by fixed block size as shown in Fig.2(b) -(d). In the proposed method, to reduce these block artifacts. Moreover, we can easily understand that the estimated accuracy of the proposed method is superior to that of three other methods.

Finally, we confirm that the merging process of the proposed method is performed adequately. Figure 3 shows the interpolated result and the wired expression of block size of the interpolated frame. In the still regions, blocks are merged into $32 \times 32$ block. In the motion regions, suitable block size is chosen. Thus, the motion can be preserved and the block artifact can be reduced.

\section{CONCLUSION}

In this paper, MC-FRUC using BMA with multi-size blocks is proposed. The proposed technique effectively reduces the block artifacts associated with the failure of ME and small block-based processing. The block artifacts by the failure of $\mathrm{ME}$ are reduced by the procedures of "zero detection" and "vector median filter" for motion vector of 8x8-block. Also, the proposed method applies the block merging, in order to estimate the motion vector with suitable block size. Experimental results show that the proposed scheme outperforms the conventional method with fixed block size in terms of the block artifacts and the accuracy of the interpolated frame images.

\section{REFERENCES}

[1] C. Cafforio, F. Rocca, and S. Tubaro, "Motion compensated image interpolation," IEEE Trans. Communication, vol.38, no.2, pp.215-222, Feb.1990.

[2] S.-H. Lee, Y.-C. Shin, S. Yang, H. -H. Moon, and R.-H. Park, "Adaptive motion-compensated interpolation rate up-conversion," IEEE Trans. Consumer Electronics, vol.48, no.3, pp.444450, Aug. 2002.

[3] S.-H. Lee, O. Kwon, and R.-H. Park, "Weighted- adaptive motion-compensated frame rate up-conversion," IEEE Trans. Consumer Electronics, vol.49, no.3, pp.485-492, Aug. 2003.

[4] G. de. Haan, P.W.A.C. Biezen, H. Huijgen, and O.A. Ojo, "True-motion estimation with 3-D recursive search block matching," IEEE Trans. Circuits and Systems for Video Tech., vol.3, no.5, pp.368-379. Oct. 1993.

[5] J.N. Youn, M.T. Sun, and C.W. Lin, "Motion estimation for high performance transcoding," IEEE Trans. Consumer Electronics, vol.44, no.3, pp.649-658, 1998.

[6] O.A. Ojo and G. de. Haan, " Robust motion-compensated video upconversion," IEEE Trans. Consumer Electronics, vol.43, no.4, pp.1045-1045, Nov. 1997.

[7] A. Nakagawa, E. Morimatsu, T. Itoh, and K. Matsuda, "Dynamic resolution conversion method for low bit-rate video transmission," IEICE Trans. Communication, vol.E84-B, no.4, April 2001

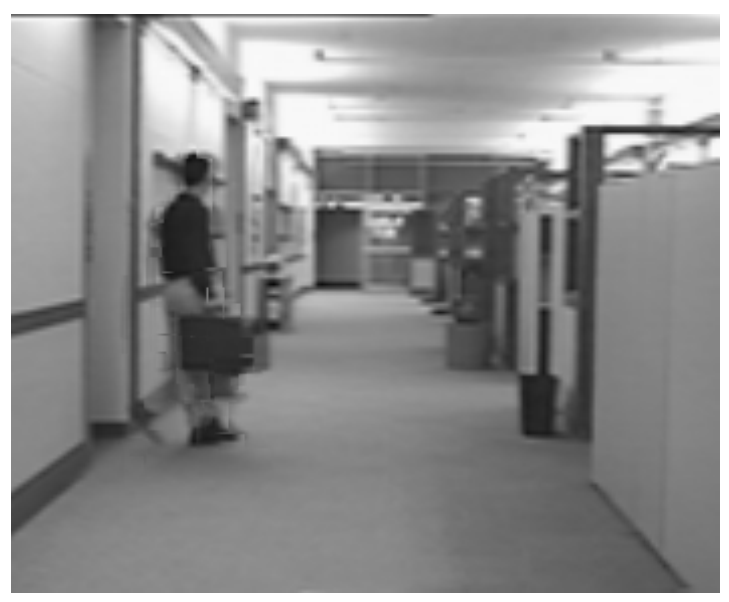

(a) The interpolated frame image is given by the proposed method

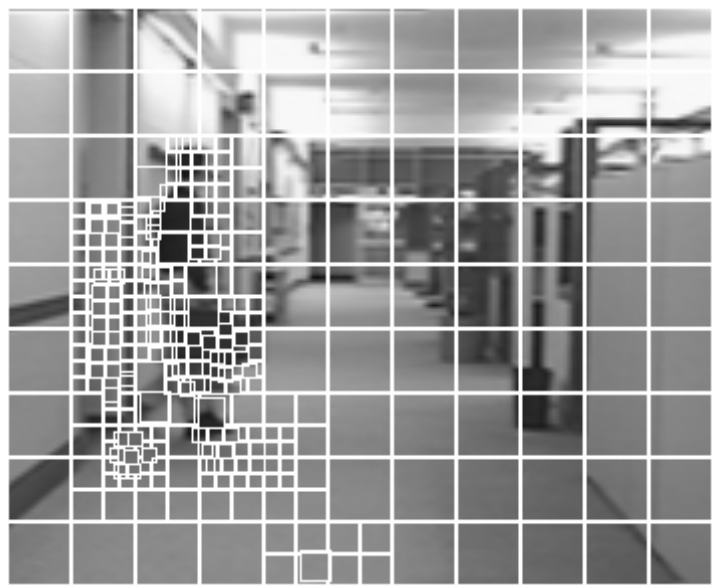

(b) The wired expression of block size of the interpolated frame

Fig.3 How to calculate of the interpolated frame image 